\title{
The ratio of the seroprevalence to the egg- positive prevalence of Schistosoma japonicum in China: a meta-analysis
}

\author{
Yao Deng ${ }^{1,2,3}$, Chen Qiu ${ }^{1,2,3}$, Huan Ding ${ }^{1,2,3}$ and Da-Bing Lu ${ }^{1,2,3^{*}}$
}

\begin{abstract}
Background: Schistosomiasis, caused by Schistosoma japonicum, remains one of the most important parasitic diseases, and detection of S. japonicum infections in humans plays a crucial role in control and treatment. However, comparisons between the parasitological and the immunological examinations in the fields of China are lacking. Therefore we performed a meta-analysis to compare the seroprevalence of Schistosoma japonicum, as determined by IHA or ELISA, with coprological prevalence, as determined by Kato-Katz, and estimate the ratio of the serological to the egg-positive prevalence in order to evaluate the potential threat of egg-negative but worm-positive schistosomiasis.
\end{abstract}

Methods: Studies published up to July 2018 on the parasitological and immunological examinations of schistosomiasis in the fields of China were searched in five databases including CNKI, WanFang, VIP, PubMed and Web of Science. The ratio of the serological to the egg-positive prevalence and its $95 \% \mathrm{Cl}$ for each study were calculated, and then point estimates and their $95 \% \mathrm{Cls}$ of pooled prevalence ratio were meta-analyzed. Subgroup meta-analyses were also performed according to potential influential factors.

Results: A total of 23 articles were included. The prevalence ratio varied from 0.57 to 48.83 for IHA to Kato-Katz and ranged from 0.38 to 13.97 for ELISA to Kato-Katz. The pooled ratio was 4.72 (95\%Cl: 3.87 5.76) for IHA to KK and 4.65 (95\%Cl: 3.50 6.17) for ELISA to KK. Subgroup analyses implied that the ratio of the serological to the egg-positive prevalence may decrease with the endemic levels. The highest prevalence ratio was observed when Kato-Katz was performed with three slides per stool or in hilly and mountainous regions.

Conclusions: The worm-determined prevalence by IHA or ELISA is 4- to 5-fold higher than the egg-determined prevalence by Kato-Katz, which implied Kato-Katz may largely underestimate the prevalence of S. japonicum in China. The degree of underestimation was greater when Kato-Katz with three slides per stool was carried out, especially in low endemic areas or in hilly and mountainous regions. Therefore, more attention should be paid to those egg-negative but worm-positive patients with the aim of final elimination of S. japonicum in China.

Keywords: Schistosoma japonicum, Kato-Katz, IHA, ELISA, Prevalence ratio

\footnotetext{
* Correspondence: Ludabing@suda.edu.cn

'Department of Epidemiology and Statistics, School of Public Health, Soochow University, Suzhou, China

${ }^{2}$ Jiangsu Key Laboratory of Preventive and Translational Medicine for Geriatric Diseases, School of Public Health, Soochow University, Suzhou, People's Republic of China

Full list of author information is available at the end of the article
}

(c) The Author(s). 2018 Open Access This article is distributed under the terms of the Creative Commons Attribution 4.0 International License (http://creativecommons.org/licenses/by/4.0/), which permits unrestricted use, distribution, and reproduction in any medium, provided you give appropriate credit to the original author(s) and the source, provide a link to the Creative Commons license, and indicate if changes were made. The Creative Commons Public Domain Dedication waiver (http://creativecommons.org/publicdomain/zero/1.0/) applies to the data made available in this article, unless otherwise stated. 


\section{Background}

Schistosomiasis, caused by blood-dwelling flukes, is one of the most prevalent parasitic diseases with over 200 million people infected, nearly 800 million people threatened and 70 million disability-adjusted life-years lost worldwide [1]. In China where Schistosoma japonicum was once highly endemic, after nearly 70 years of control a great progress has been made [2]. For example, the number of infected people had declined from 11.6 million in the mid-1950s to nearly 77.2 thousand in 2015 $[3,4]$. By the end of 2015, out of 453 endemic counties (city, district), 343 and 110 had achieved criteria of transmission interruption and of transmission control, respectively. The overall objective of the Mid- and Long-term National Plan for Prevention and Control of Schistosomiasis of China (2004-2015) has been achieved on schedule [5]. Based on above achievements, therefore, a new goal-to interrupt transmission by 2020 and then to completely eliminate the disease across China by 2025-for the next 10 years was proposed in 2015 [6]. However, due to the complex lifecycle of Schistosoma japonicum and many natural and social factors in affecting the transmission and spread of schistosomiasis, schistosomiasis elimination still faces serious challenges. In addition, no effective vaccine is available and praziquantel is the only recommended drug by WHO for the treatment of schistosomiasis [7, 8]. Therefore, accurately assessing the real prevalence of Schistosoma japonicum infections in humans through simple and sensitive diagnostic tests is very essential in China [9].

There are currently various diagnostics for $S$. japonicum infection in humans, including direct parasitological tests, immunological techniques and molecular diagnosis [10]. Direct parasitological tests, such as the Kato-Katz thick smear technique (Kato-Katz or KK), are the earliest diagnosis used for identification of schistosomiasis and are still widely applied nowadays [11]. However, with the continual decrease of the prevalence and intensity of $S$. japonicum infection, parasitological tests are not sensitive and accurate enough to estimate the real prevalence, especially in low endemic regions [12-14]. Immunological techniques, including the indirect hemagglutination assay (IHA) and the enzyme-linked immunosorbent assay (ELISA), have become more popular in schistosome detection as they are more sensitive, rapid and easy to perform $[15,16]$. A number of studies have been conducted to evaluate the effectiveness of different diagnostic assays either in the laboratory or in the field. However, the different or controversial results were among these findings. A meta-analysis on the immunodiagnostic efficacies of IHA and ELISA in field settings performed by Wang et al. [17] revealed that IHA is superior to ELISA, but the research by Wang et al. [18] showed that ELISA is better than IHA. Three other meta-analyses [19-21] were used to assess the accuracy of IHA, ELISA and the dipstick dye immunoassay (DDIA), respectively. All the above articles were mainly aimed at immunological tests. However, comparisons between parasitological and immunological examinations of the same village-based subjects in the field of China have not yet been conducted. The neglect of egg-negative but worm-positive schistosomiasis, especially in low endemic areas, may influence control effects and thus hinder the elimination of schistosomiasis [22]. Therefore, we performed this meta-analysis to compare the seroprevalence of Schistosoma japonicum, as determined by IHA or ELISA, with coprological prevalence, as assessed using Kato-Katz, and estimate the ratio of the serological to the egg-positive prevalence. The main purpose was to evaluate the potential threat of egg-negative but worm-positive schistosomiasis.

\section{Methods}

\section{Study protocol and registration}

The protocol for this study was developed prospectively and registered in the international Prospective Register of Systemic Reviews (PROSPERO) online database (https:// www.crd.york.ac.uk/PROSPERO, with registered number CRD42017067941) on June 6, 2017. The protocol is provided as Supporting Information (see Additional file 1).

\section{Search strategy}

A database search through July 2018 was performed to identify relevant studies regarding the comparison between seroprevalence of S. japonicum determined by IHA or ELISA and coprological prevalence with Kato-Katz. We aimed to include all published studies in Chinese or English. Three Chinese literature databases, including China National Knowledge Infrastructure (CNKI), WanFang Database and Chinese Scientific Journal Database (VIP), and two English literature databases, including PubMed and Web of Science, were searched for data pertaining to the prevalence rate of $S$. japonicum determined by three diagnostic tests in field surveys in China. We used the following search terms (the corresponding Chinese keyword in Pinyin (phoneticism) was given between brackets): "schistosoma", "schistosomiasis", "bilharzia", "bilharziasis" (xuexichong) in combination with "Kato" and "IHA" or "ELISA". We did not contact authors of original studies for additional information. No attempt was made to identify unpublished studies.

\section{Study selection}

An initial screen of identified titles and abstracts was performed by YD and CQ. A second screen of full-text articles was then conducted if the studies were found suitable for inclusion. Studies to qualify the following criteria were included: (1) field surveys; (2) targeted subjects included residents from endemic villages, and received both a fecal examination with Kato-Katz and a 
serological examination with IHA or ELISA, simultaneously; (3) a survey in one village, or more villages of the same endemic level, could be taken as a study; (4) one stool sample from each participant was provided; (5) data on numbers of the positive and the total examined were provided, or could be obtained by formula. Studies to exclude were: (1) about animals or from laboratories; (2) in the same village and repeated periods; (3) no eggs found in a stool specimen; (4) about floating populations, such as fishermen and boatmen; (5) full texts unavailable.

\section{Data extraction and quality assessment}

The detailed features of each eligible study were extracted using a purpose-built data-collection excel form. Information was recorded as follows: study characteristics (last name of the first author, year of publication, period of study, location); characteristics of targeted villages (the recorded prevalence level and the type of endemic area); study methodology (the fecal and serological examinations used); numbers of the positive and the total of persons assessed. Information about the endemic level or type of the targeted villages was extracted from relevant articles if such data were not provided in the eligible studies.

Bias in data collection was reduced through the involvement of $\mathrm{YD}$ and $\mathrm{CQ}$, who independently evaluated the quality of studies. In cases of disagreement, HD was consulted in order to resolve the problem.

\section{Data analyses}

The ratio of the serological to the egg-positive prevalence and its $95 \% \mathrm{CI}$ for each study were first calculated. We then meta-analyzed the point estimate of pooled ratio and the corresponding 95\% CI [23, 24].

In all analyses, Cochran's $\mathrm{Q}$ test (significance level at $P$ $<0.10)$ and $\mathrm{I}^{2}$ statistics were applied to measure the heterogeneity [25]. The $\mathrm{I}^{2}$ statistics is a quantitative measure of inconsistency across studies, with values of 25, 50 and $75 \%$ corresponding to low, moderate, and high degrees of heterogeneity, respectively [26]. In the absence of observed heterogeneity between studies, the fixed effects model should be used for the data analysis; otherwise, the random effects model should be selected [27, 28]. We conducted subgroup analyses stratified by the endemic level or the type of targeted villages, or the number of slides used per stool, to evaluate the influence of these factors on outcomes.

Potential publication bias of studies was assessed with both Begg rank correlation test and Egger linear regression test $[29,30]$. To test the robustness of the pooled ratio, we conducted a sensitivity analysis by omitting one study or publication at a time, and each time we calculated the pooled estimate for the remaining ones [31].
Extracted data were entered into Microsoft Office Excel 2013 and all statistical analyses were performed with Stata 14.0. $P<0.05$ was considered statistically significant, except where otherwise specified. All statistical tests were two-sided. The PRISMA (Preferred Reporting Items for Systematic reviews and Meta-Analyses) statement was used as a guide in this paper [32].

\section{Results}

\section{Literature search}

Figure 1 contains a flow chart that describes search results and selection strategy for the studies included in this meta-analysis. We retrieved 471 published articles through five databases, of which 192 articles were excluded when taking duplication into consideration. After the initial screening of titles and abstracts, a further 216 articles were excluded. Consequently, we identified 63 potentially relevant publications for full-text review, of which 40 articles were excluded according to the inclusion criteria. Finally, a total of 23 articles were enrolled in this meta-analysis, with 11 articles about IHA [33-44], six about ELISA [45-50] and six about both IHA and ELISA [51-55].

\section{Study characteristics}

Table 1 and Table 2 show the characteristics of the final 23 publications, which covered six provinces, namely Jiangsu, Anhui, Hunan, Hubei, Jiangxi and Yunnan provinces. The years of the studies performed and published ranged from 1990 to 2012 and from 1992 to 2015, respectively. The number of participants ranged from 101 to 1956 with IHA, 68 to 1024 with ELISA and 68 to 1956 with Kato-Katz.

\section{Main analysis}

As seen in Fig. 2, the prevalence ratio of IHA to Kato-Katz varied from 0.57 to 48.83 , with substantial heterogeneity among studies $\left(x^{2}=713.34, P<0.001 ; I^{2}=93.7 \%\right)$. The prevalence ratio of ELISA to Kato-Katz ranged from 0.38 to 13.97 , with substantial heterogeneity among studies $\left(x^{2}=266.77, P<0.001 ; I^{2}=91.0 \%\right)$. See Fig. 3 . The pooled prevalence ratio was 4.72 (95\%CI: 3.87 5.76) for IHA to Kato-Katz and 4.65 (95\%CI: 3.50 6.17) for ELISA to Kato-Katz when calculated with the random-effects model.

\section{Subgroup analyses}

Table 3 shows the results of subgroup analyses stratified by the endemic level, the number of slides used per stool sample and the type of endemic area. Due to the high heterogeneity among studies within most subgroups, pooled ratios for each subgroup were calculated using the random-effects model. In terms of endemic levels, the pooled ratio of IHA to Kato-Katz 


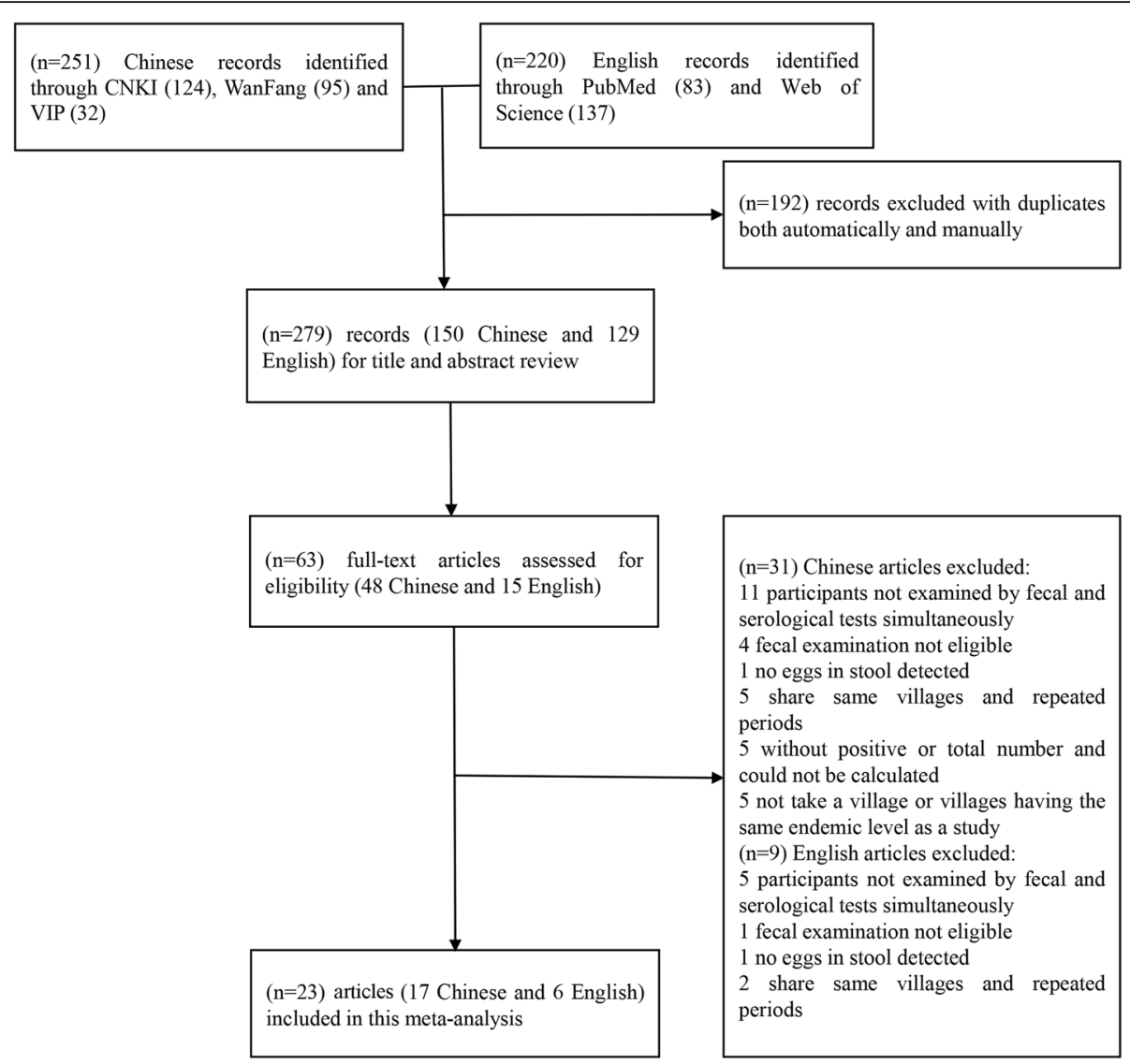

Fig. 1 Flow chart of study selection

showed the highest estimate in the middle endemic areas (RR: $4.99,95 \% \mathrm{CI}: 3.39 \sim 7.34$ ) and the lowest in high endemic (RR: 4.05, 95\%CI: 2.93 5.60). A downtrend was observed in the pooled ratios of ELISA to Kato-Katz (Low endemic areas: 6.06, 95\%CI: 2.22 16.58; Middle: 5.92, 95\%CI: 2.74 12.80; High: 3.06, 95\%CI: 2.09 4.50). In terms of the number of slides per stool in Kato-Katz, the highest ratio of IHA to Kato-Katz (PRR: 5.03, 95\%CI: 4.05 6.25) was obtained for three slides per stool specimen, whereas the lowest ratio (PRR: 2.47, 95\%CI: 1.70 3.57) was obtained for over four slides per stool. The ratio of ELISA to Kato-Katz increased with the number of slides used (2 slides: 4.11, 95\%CI: 1.76 9.61; 3 slides: 4.41, 95\%CI: $3.20 \sim 6.08$; $\geq 4$ slides: 10.38, 95\%CI: 5.97 18.05).

Subgroup analyses showed the highest pooled estimates in the hilly and mountainous regions (IHA to KK: 15.86, 95\%CI: 1.47 171.60; ELISA to KK: 7.20 , 95\%CI: 3.33 15.57). The lowest was in plains with water network areas for IHA to Kato-Katz (RR: 3.64, 95\%CI: 2.64 5.00) and in marshlands and lake regions for ELISA to Kato-Katz (RR: 3.73, 95\%CI: 2.48 5.60).

\section{Sensitivity analyses}

Sensitivity analyses presented that all single-study-omitted and single-paper-omitted estimates were within the 95\% CIs of their respective overall ratios except one paper (see Additional files 2, 3, 4 and 5). This suggested that the pooled ratios were not substantially modified by any single study, or by any single paper except Huang et al. [45]. The stability of such results validated the rationality and reliability of our analyses.

\section{Publication bias}

There was no evidence of publication bias, as suggested by Begg rank correlation test $(\mathrm{z}=1.18, P=0.237$ for IHA and $\mathrm{z}=0.54, P=0.591$ for ELISA) and Egger linear regression test (the bias coefficients $\mathrm{b}=2.64,95 \% \mathrm{CI}$ : -0.17 5.46, $t=1.89, P=0.065$ for IHA and $\mathrm{b}=-0.69$, 95\%CI: $-3.45 \sim 2.07, t=-0.52, P=0.610$ for ELISA).

\section{Discussion}

Egg-negative but worm-positive schistosomiasis may be a potential threat for achieving the goal of schistosomiasis elimination [22]. Therefore, accurate detection of Schistosoma japonicum infections in humans plays a 


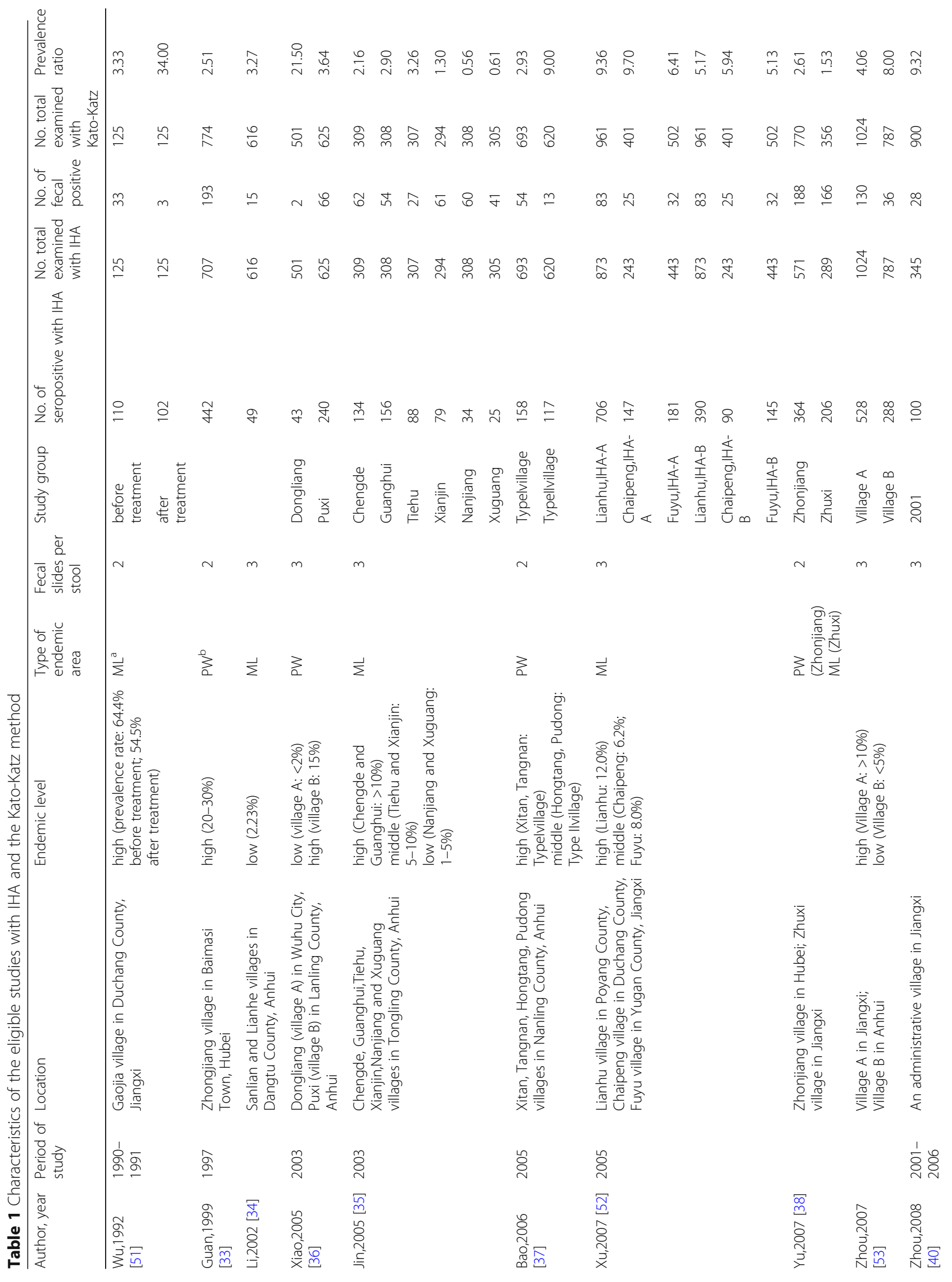




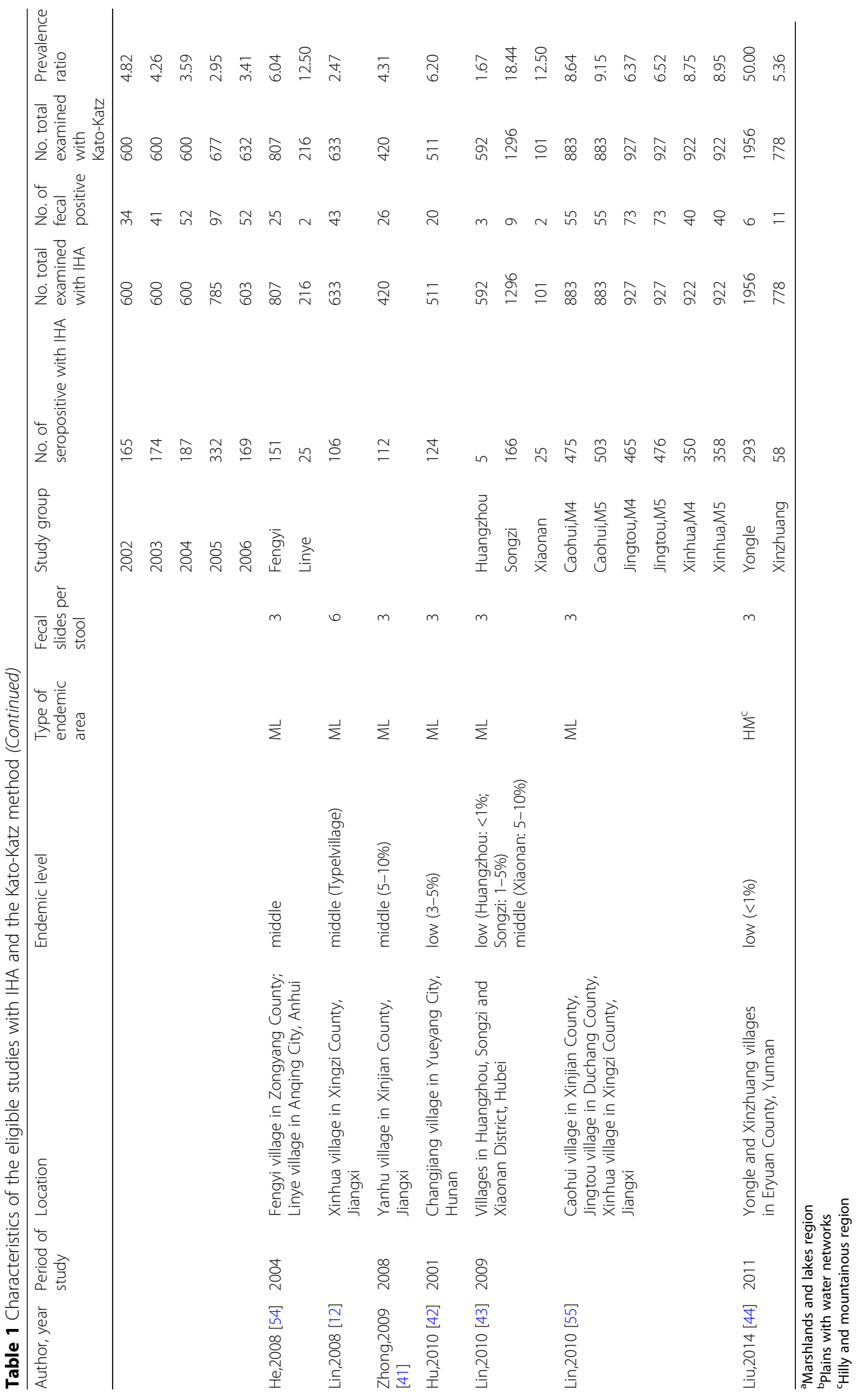




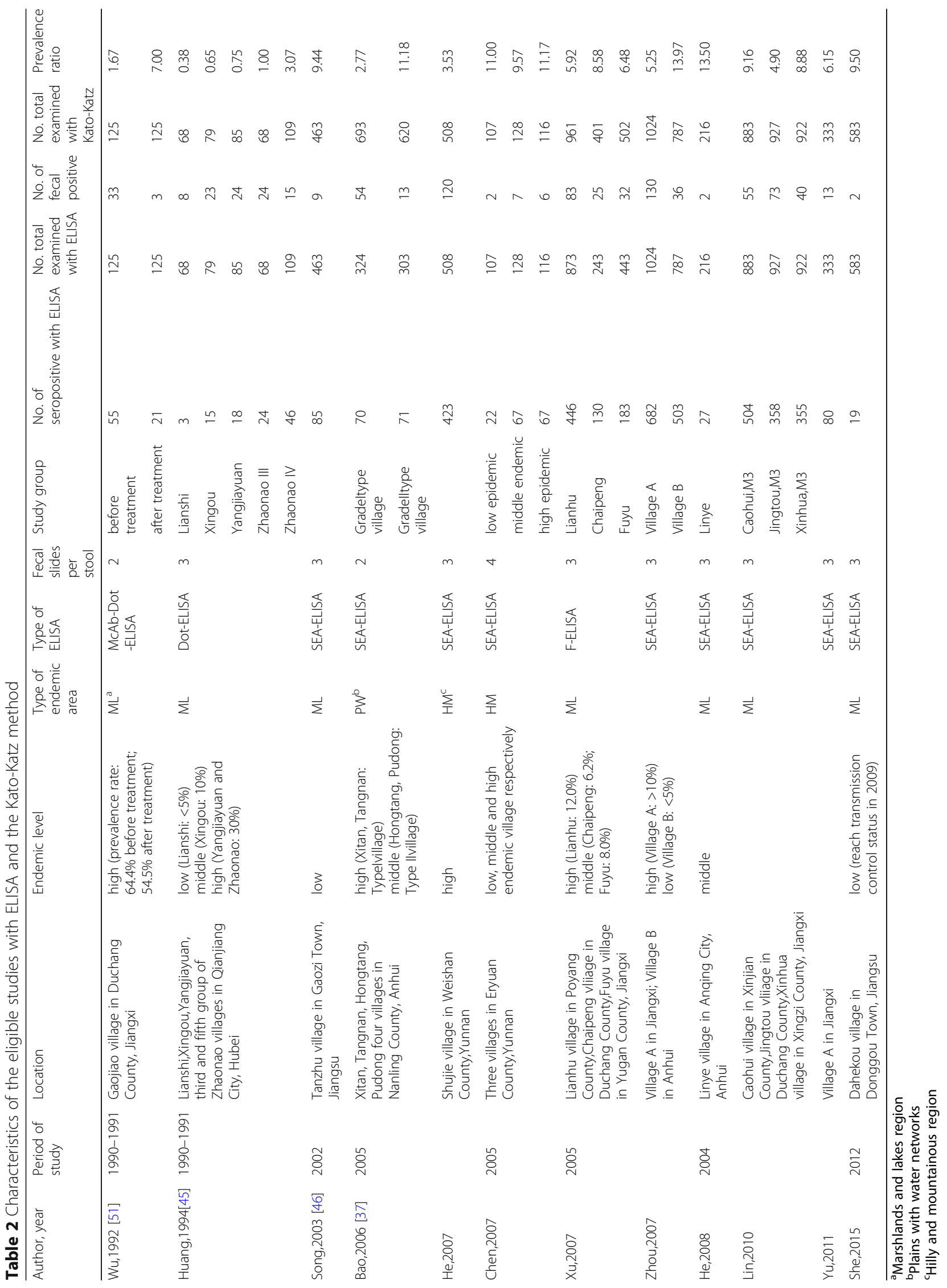




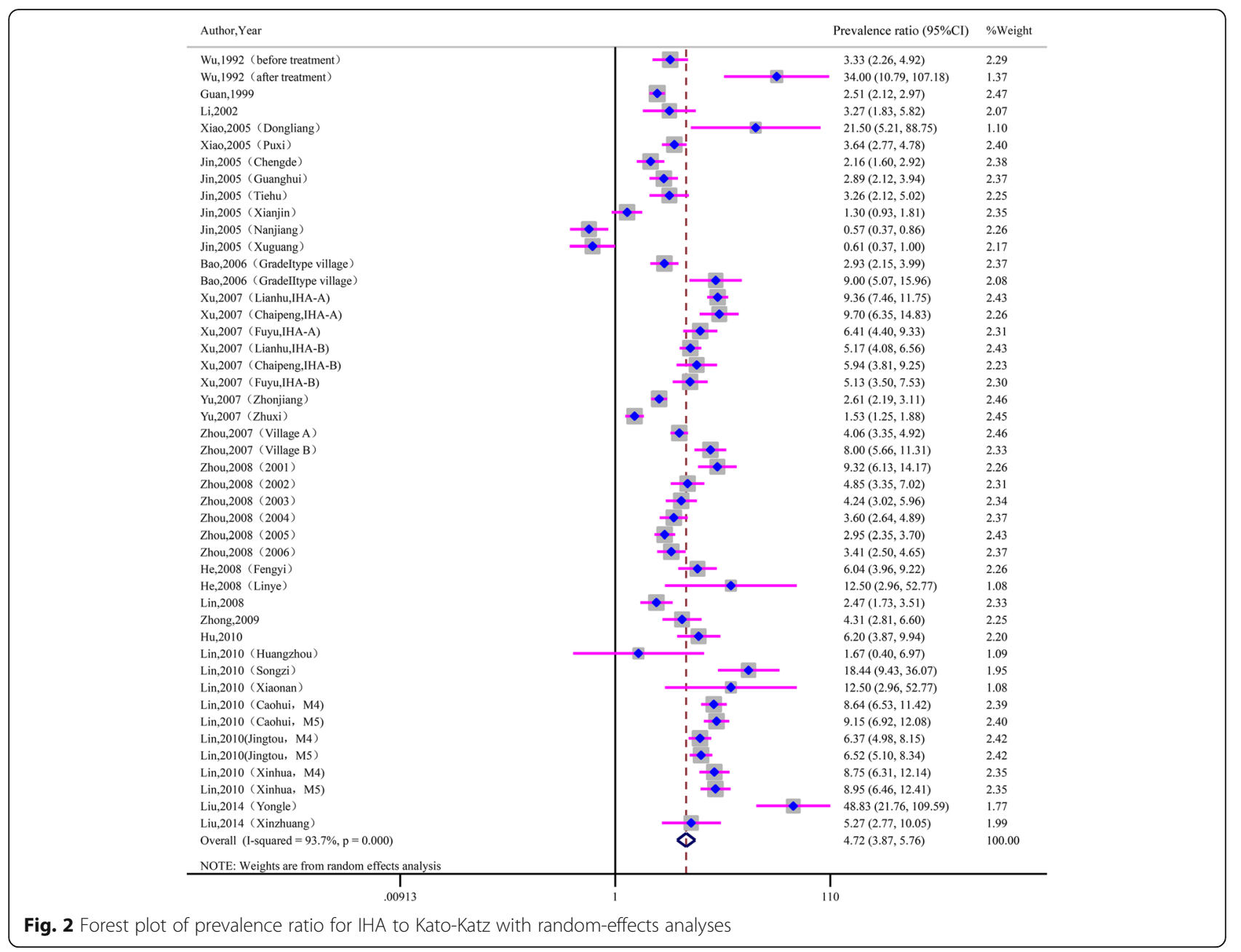

crucial role in the process of control and treatment [56]. There were also a number of other publications exploring the differences of parasitological and immunological diagnostic assays in field situations in China, but in those research Kato-Katz was only applied to human individuals after they had been tested positive with immunodiagnostic method, not qualifing the criteria of inclusion in our research [13, 56-69]. We here included only 23 articles in the current meta-analysis [33-55]. The findings of this meta-analysis showed that the prevalence by IHA or ELISA is 4- to 5-fold higher than the prevalence by Kato-Katz, which indicated that Kato-Katz clearly underestimated the prevalence of $S$. japonicum in China. Subgroup analyses suggested that the ratio of the serological to the egg-positive prevalence decreased with the endemic levels. A highest prevalence ratio was obtained when the number of slides per stool was three and was in the hilly and mountainous regions.

Compared with the egg-determined prevalence by a single stool examination, the worm-determined prevalence by IHA or ELISA were about 4-5 times higher, indicating the existence of substantial inconsistencies between two types of examinantions. Multiple stool examinations with Kato-Katz can increase the positive rate and obtain more accurate results, but it is impractical in the field $[10,70]$. The Kato-Katz test is most widely used and the misdiagnosis or even absence of eggs can be quite common in the field, especially in low endemic regions $[40,71,72]$. Therefore, it is obvious that the positive prevalence with one stool examination is much lower than with IHA and ELISA.

Although the overall seroprevalence was about 4- to 5 -fold higher than the pooled coprological prevalence, the prevalence ratio per study varied from 0.57 to 48.83 for IHA to KK and ranged from 0.38 to 13.97 for ELISA to KK. Several explanations could account for the substantial heterogeneity among studies. First, 14 eligible publications provided more than one group of studies and these studies could not be combined directly [35-38, 40, 43-45, 47, 51-55]. Second, study regions and periods varied in epidemiological settings, such as types of endemic areas, recorded prevalence level and transmission intensity. Third, different diagnostic reagents and the number of slides per stool 


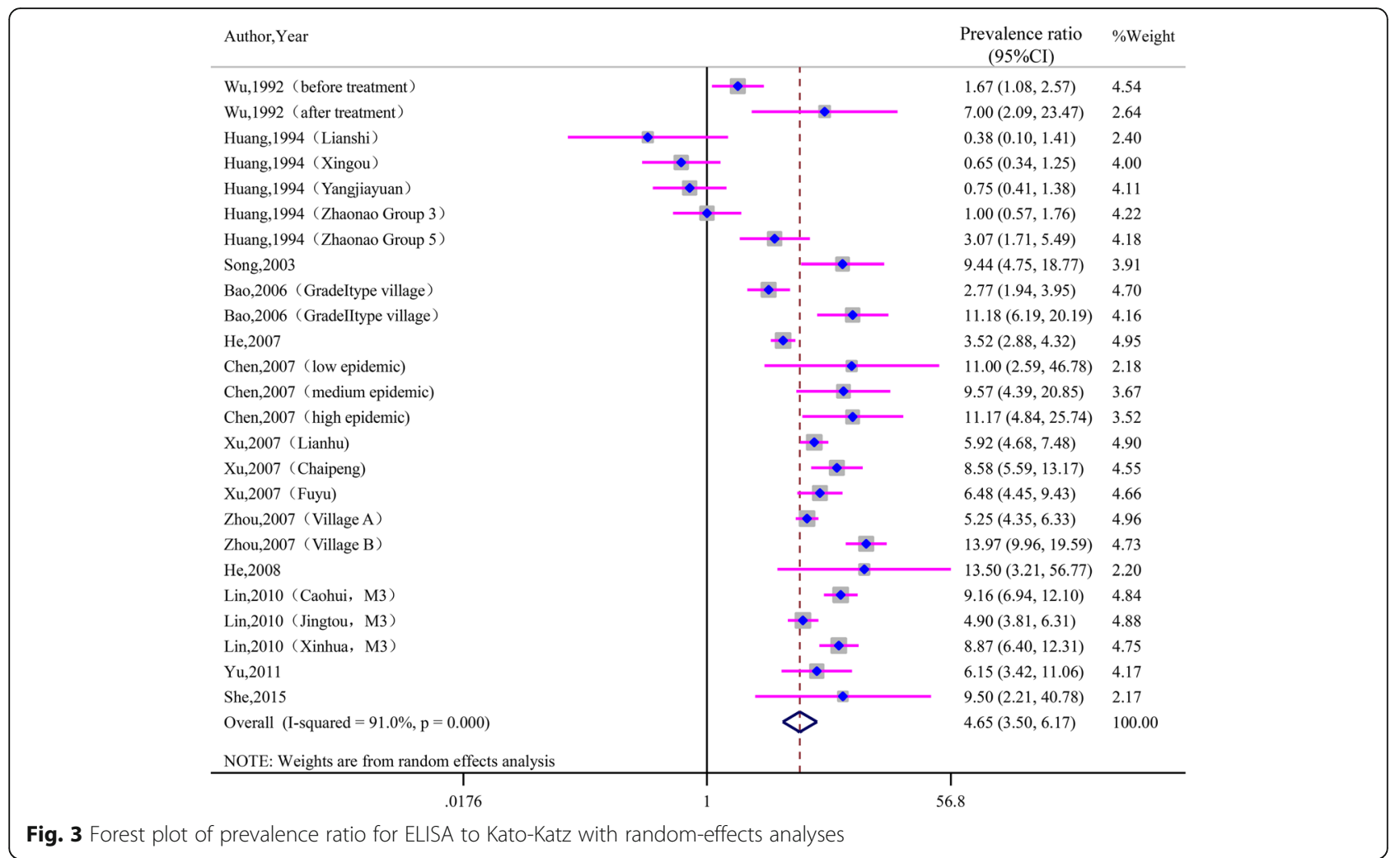

may also cause the discrepancies. Fourth, sample sizes among included studies differed greatly, ranging from 68 to 1956. Finally, there are several cases which should be noted. The prevalence ratio of IHA to Kato-Katz was 50 in Yongle Village [44] and 34 after mass chemotherapy in Gaojia Village [51], which were much higher than the others. The infection rate in
Yongle village was very low (0.8\%) with Kato-Katz, leading to an underestimation of true infections [44]. In Gaojia village after 3 months of treatment the number of infections with Kato-Katz may decrease sharply but the antigen level of IHA in serum should be still very high, thus resulting in the high ratio [51]. When comparing Kato-Katz and ELISA, most articles

Table 3 Pooled estimates of prevalence ratio by potential influential factors with meta-analysis

\begin{tabular}{|c|c|c|c|c|c|c|c|c|c|c|}
\hline \multirow[t]{2}{*}{ Group } & \multicolumn{5}{|c|}{$\mathrm{IHA}$ to $\mathrm{KK}$} & \multicolumn{5}{|c|}{ ELISA to KK } \\
\hline & $\begin{array}{l}\text { No. of } \\
\text { papers }\end{array}$ & $\begin{array}{l}\text { No. of } \\
\text { studies }\end{array}$ & $\begin{array}{l}\text { Prevalence } \\
\text { ratio }(95 \% \mathrm{Cl})\end{array}$ & $P_{\text {heterogeneity }}$ & $\overline{I^{2}(\%)}$ & $\begin{array}{l}\text { No. of } \\
\text { papers }\end{array}$ & $\begin{array}{l}\text { No. of } \\
\text { studies }\end{array}$ & $\begin{array}{l}\text { Prevalence } \\
\text { ratio }(95 \% \mathrm{Cl})\end{array}$ & $P_{\text {heterogeneity }}$ & $P^{2}(\%)$ \\
\hline Total & 17 & 46 & $4.72(3.87,5.76)$ & 0.000 & 93.7 & 12 & 25 & $4.65(3.50,6.17)$ & 0.000 & 91.0 \\
\hline \multicolumn{11}{|l|}{ Endemic level } \\
\hline Low & 7 & 10 & $4.89(1.92,12.43)$ & 0.000 & 95.9 & 5 & 5 & $6.06(2.22,16.58)$ & 0.000 & 84.2 \\
\hline Middle & 7 & 12 & $4.99(3.39,7.34)$ & 0.000 & 86.6 & 5 & 6 & $5.92(2.74,12.80)$ & 0.000 & 89.0 \\
\hline High & 7 & 10 & $4.05(2.93,5.60)$ & 0.000 & 91.5 & 7 & 10 & $3.06(2.09,4.50)$ & 0.000 & 88.7 \\
\hline \multicolumn{11}{|l|}{ Number of slides per stool } \\
\hline 2 & 4 & 7 & $3.45(2.37,5.03)$ & 0.000 & 89.2 & 2 & 4 & $4.11(1.76,9.61)$ & 0.000 & 88.2 \\
\hline 3 & 12 & 38 & $5.03(4.05,6.25)$ & 0.000 & 91.7 & 9 & 18 & $4.41(3.20,6.08)$ & 0.000 & 90.6 \\
\hline$\geq 4$ & 1 & 1 & $2.47(1.70,3.57)$ & - & - & 1 & 3 & $10.38(5.97,18.05)$ & 0.965 & 0.0 \\
\hline \multicolumn{11}{|l|}{ Type of endemic area } \\
\hline Marshlands and lakes region & 11 & 30 & $4.60(3.45,6.14)$ & 0.000 & 93.8 & 7 & 16 & $3.73(2.48,5.60)$ & 0.000 & 90.7 \\
\hline Plains with water networks & 4 & 6 & $3.64(2.64,5.00)$ & 0.000 & 82.2 & 1 & 2 & $5.45(1.37,21.66)$ & 0.000 & 93.3 \\
\hline Hilly and mountainous regions & 1 & 2 & $15.86(1.47,171.60)$ & 0.000 & 95.2 & 2 & 4 & $7.20(3.33,15.57)$ & 0.005 & 76.5 \\
\hline
\end{tabular}


applied the routine ELISA method to detect the antibodies, whereas two articles $[45,51]$ applied the Dot-ELISA method to detect the CAg in serum. However, the results between two papers were controversial. All these factors may lead to such differences among these studies.

Subgroup analyses, based on limited numbers of papers, revealed that the prevalence ratio decreased with the increase level of endemic degrees, although the ratio of IHA to ELISA was slightly higher in middle endemic villages than in low endemic villages. This may be mainly related to a high false negative rate for egg detection in stool in low endemic areas, particularly after widespread chemotherapy [73, 74].

Repeated and multiple stool examinations can provide more accurate results, but it is quite time-consuming and strenuous in the filed [10,70]. As different stool specimens can not be compared directly, we only included articles in which a single stool examination was carried out. The findings showed that the highest prevalence ratio of IHA to Kato-Katz was 5.03 (95\%CI: 4.05 6.25) with three slides per stool, and of ELISA to Kato-Katz was 10.38 (95\%CI: 5.97 18.05) with $\geq 4$ slides per stool. However, it is noted that only one article [47] provided data of fecal examinations with equal or more than four slides per stool specimen. So the uptrend of the prevalence ratio of ELISA to Kato-Katz with the increase of numbers of slides per stool was suspected. A relative high ratio was observed when examined with Kato-Katz with three slides per stool in our meta-analysis. Indeed, Lin et al. [75] once reported that the rates of underestimation with three slides per stool specimen could reach $40.98 \sim 50.80 \%$, especially in low endemic areas.

There were only three articles involved in hilly and mountainous regions $[44,47,48]$ with one on IHA to Kato-Katz and two on ELISA to Kato-Katz. We believed that the patients in Yongle village could, because of the low infection rate by the Kato-Katz method, be largely missed [44]. Overall, the current meta-analysis showed the highest prevalence ratio in hilly and mountainous regions. This may provide support for further schistosomiasis control among different endemic areas.

However, there are some disadvantages for immunological tests. The main one is the relatively high false-positive rate, as they can not discriminate active infection from past infection [76-78]. The false-positive rates of IHA and ELISA were reported to be 0.062 to 0.643 and 0.157 to 0.755 , respectively [17]. In this meta-analysis, two articles $[45,51]$ applied the Dot-ELISA method to detect the CAg in serum. The negative reversal rate of egg-positive patients was $66.7 \%$ with McAb-Dot-ELISA and 0 with IHA after 3 months of treatment [51]. However, research also showed that most patients turned negative with
IHA after effective and periodical treatment for 3 years or more [16]. After one to 2 years of treatment, the negative reversal rate was nearly $60 \%$ in the ELISA assay, and most patients turned negative in the Dot-ELISA test [16]. Overall, the Dot-ELISA assay appears to be more efficient than the routine IHA or ELISA. The false-positive rate of immunological tests is also a limitation of this paper, partly leading to an inflated prevalence ratio of IHA to Kato-Katz or of ELISA to Kato-Katz.

\section{Conclusions}

In summary, the worm-determined prevalence by IHA or ELISA was about 4- to 5-fold higher than the egg-determined prevalence by Kato-Katz, which implied that the Kato-Katz method may largely underestimate the infection prevalence of $S$. japonicum in the field. The degree of underestimation became worse when being examined by Kato-Katz with three slides per stool, especially in low endemic areas or in hilly and mountainous regions. Such significant difference between the fecal and the serological examinations may lead to the existence of a sizable population of "egg-negative but worm-positive schistosomiasis". Therefore, more attention should be paid to those infected humans with the aim of final elimination of S. japonicum in China.

\section{Additional files}

Additional file 1: PROSPERO (CRD42017067941): A meta-analysis of the ratio of seroprevalence to egg-positive prevalence of Schistosoma japonicum in China. (PDF $117 \mathrm{~kb}$ )

Additional file 2: Sensitivity analyses for IHA to Kato-Katz by singlestudy-omitted. (TIF $447 \mathrm{~kb}$ )

Additional file 3: Sensitivity analyses for ELISA to Kato-Katz by singlestudy-omitted. (TIF $356 \mathrm{~kb}$ )

Additional file 4: Sensitivity analyses for IHA to Kato-Katz by singlepaper-omitted. (TIF $247 \mathrm{~kb}$ )

Additional file 5: Sensitivity analyses for ELISA to Kato-Katz by singlepaper-omitted. (TIF $214 \mathrm{~kb}$ )

\section{Abbreviations}

Cl: Confidence intervals; CNKI: China National Knowledge Infrastructure; DDIA: Dipstick dye immunoassay; ELISA: Enzyme-linked immunosorbent assay; IHA: Indirect hemagglutination assay; KK (Kato-Katz): Kato-Katz thick smear technique or the Kato-Katz method; PRISMA: Preferred reporting items for systematic reviews and meta-analyses; VIP: Chinese Scientific Journal Database

\section{Funding}

This work was funded by the Jiangsu Provincial Project of Invigorating Health Care through Science, Technology and Education and the National Science Foundation of China (No.81273141).

Availability of data and materials

All the datasets generated and/or analyzed during the current study are included in this article.

\section{Authors' contributions}

YD and DBL conceived of and designed the study. YD and CQ carried out the screen of the literature and data extraction, and checked by HD. YD 
analyzed the results with help of HD and DBL. YD drafted the manuscript, and $C Q, H D$ and $D B L$ revised the manuscript. All authors read and approved the final manuscript.

\section{Ethics approval and consent to participate}

Not applicable.

\section{Consent for publication}

Not applicable.

\section{Competing interests}

The authors declare that they have no competing interests.

\section{Publisher's Note}

Springer Nature remains neutral with regard to jurisdictional claims in published maps and institutional affiliations.

\section{Author details}

'Department of Epidemiology and Statistics, School of Public Health, Soochow University, Suzhou, China. ${ }^{2}$ Jiangsu Key Laboratory of Preventive and Translational Medicine for Geriatric Diseases, School of Public Health, Soochow University, Suzhou, People's Republic of China. ${ }^{3}$ Key Laboratory of National Health and Family Planning Commission on Parasitic Disease Control and Prevention, Jiangsu Provincial Key Laboratory on Parasite and Vector Control Technology, Jiangsu Institute of Parasitic Diseases, Wuxi, Jiangsu Province, China.

Received: 16 November 2017 Accepted: 9 August 2018 Published online: 15 August 2018

\section{References}

1. Gray DJ, Mcmanus DP, Li YS, Williams GM, Bergquist R, Ross AG. Schistosomiasis elimination: lessons from the past guide the future. Lancet Infect Dis. 2010:10(10):733-6.

2. Chen M. Assessment of morbidity due to Schistosoma japonicum infection in China. Infect Dis Poverty. 2014;3(1):6.

3. Zhou XN, Wang LY, Chen MG, Wu XH, Jiang QW, Chen XY, Zheng J, Utzinger J. The public health significance and control of schistosomiasis in China--then and now. Acta Trop. 2005;96(3):97-105.

4. Zhang LJ, Zhi-Min XU, Qian YJ, Dang H, Shan LV, Jing XU, Shi-Zhu LI, Zhou XN. Endemic status of schistosomiasis in People's Republic of China in 2015 Chinese J Schistosomiasis Control. 2016;

5. Zhou XN. Implementation of precision control to achieve the goal of schistosomiasis elimination in China. Chinese J Schistosomiasis Control. 2016;28(1):1

6. Sun LP, Wang W, Hong QB, Li SZ, Liang YS, Yang HT, Zhou XN. Approaches being used in the national schistosomiasis elimination programme in China: a review. Infect Dis Poverty. 2017;6(1):55.

7. Egesa M, Hoffmann KF, Hokke CH, Yazdanbakhsh M, Cose S. Rethinking schistosomiasis vaccine development: synthetic vesicles. Trends Parasitol. 2017;33(12):918-21.

8. Neves BJ, Andrade CH, Cravo PV. Natural products as leads in schistosome drug discovery. Molecules. 2015;20(2):1872

9. Song LG, Wu XY, Sacko M, Wu ZD. History of schistosomiasis epidemiology, current status, and challenges in China: on the road to schistosomiasis elimination. Parasitol Res. 2016:115(11):4071-81.

10. Zhou $Y$, Zheng $H$, Jiang Q. A diagnostic challenge for schistosomiasis japonica in China: consequences on praziquantel-based morbidity control. Parasit Vectors. 2011;4(1):1-7.

11. Weerakoon KG, Gobert GN, Cai P, Mcmanus DP. Advances in the diagnosis of human schistosomiasis. Clin Microbiol Rev. 2015;28(4):939-67.

12. Lin DD, Liu JX, Liu YM, Hu F, Zhang YY, Xu JM, Li JY, Ji MJ, Bergquist R, Wu $\mathrm{GL}$. Routine Kato-Katz technique underestimates the prevalence of Schistosoma japonicum: a case study in an endemic area of the People's Republic of China. Parasitol Int. 2008:57(3):281.

13. Xin X, Zhu HP, Yu CH, Xu XJ, Li RD, Qiu J. A Bayesian approach to estimate the prevalence of Schistosomiasis japonica infection in the Hubei Province Lake regions, China. Int J Environ Res Public Health. 2013;10(7):2799.

14. Zhu HQ, Xu J, Zhu R, Cao CL, Bao ZP, Yu O, Zhang LJ, Xu XL, Feng Z, Guo JG. Comparison of the miracidium hatching test and modified Kato-Katz method for detecting Schistosoma japonicum in low prevalence areas of China. Southeast Asian J Trop Med Public Health. 2014:45(1):20-5.

15. Sun K, Xing W, Yu X, Fu W, Wang Y, Zou M, Luo Z, Xu D. Recombinase polymerase amplification combined with a lateral flow dipstick for rapid and visual detection of Schistosoma japonicum. Parasit Vectors. 2016 9(1):476.

16. Zhu YC. Immunodiagnosis and its role in schistosomiasis control in China: a review. Acta Trop. 2005:96(2-3):130.

17. Wang W, Li Y, Li H, Xing Y, Qu G, Dai J, Liang Y. Immunodiagnostic efficacy of detection of Schistosoma japonicum human infections in China: a meta analysis. Asian Pac J Trop Med. 2012;5(1):15-23.

18. Wang $X Y$, Yang K. Serological diagnosis methods of schistosomiasis japonica at different prevalence: a meta-analysis. Chin J Schisto Control. 2016;1:18-25.

19. Wang LY, Wang YB, Wei DD. Efficiency of indirect hemagglutination assay (IHA) for the diagnosis of Schistosoma japonicum human infections: a metaanalysis. J Pathogen Biol. 2017;2:155-9.

20. Ye XF, Wang J, Cao PP, Zhuang GH, Yang XJ, Qian CY. Effectiveness of an enzyme-linked immunosorbent assay (ELISA) at diagnosing schistosomiasis in humans: a meta-analysis. J Pathogen Biol. 2016:1:50-4.

21. Qian $Y L$, Wang W, Hong QB. Performance of a dipstick dye immunoassay to diagnose schistosomiasis: a meta-analysis. J Pathogen Biol. 2014;9(6):538-41.

22. Colley DG, Andros TS, Campbell CH. Schistosomiasis is more prevalent than previously thought: what does it mean for public health goals, policies, strategies, guidelines and intervention programs? Infect Dis Poverty. 2017; 6(1):63.

23. Heller S, Mathieu C, Kapur R, Wolden ML, Zinman B. A meta-analysis of rate ratios for nocturnal confirmed hypoglycaemia with insulin degludec vs. insulin glargine using different definitions for hypoglycaemia. Diabet Med. 2015;106(4):478-87.

24. Kunz LM, Normand SL, Sedrakyan A. Meta-analysis of rate ratios with differential follow-up by treatment arm: inferring comparative effectiveness of medical devices. Stat Med. 2015;34(21):2913-25.

25. Higgins JP, Thompson SG. Quantifying heterogeneity in a meta-analysis. Stat Med. 2002;21(11):1539.

26. Higgins JPT, Thompson SG, Deeks JJ, Altman DG. Measuring inconsistency in meta-analyses. Br Med J. 2003;327(7414):557-60.

27. Dersimonian R, Laird N. Meta-analysis in clinical trials. Control Clin Trials. 1986:7(3):177-88.

28. Mantel N, Haenszel W. Statistical aspects of the analysis of data from retrospective studies of disease. Jnci J Natl Cancer Inst. 1959:22(4):719-48.

29. Begg CB, Mazumdar M. Operating characteristics of a rank correlation test for publication bias. Biometrics. 1994;50(4):1088.

30. Egger M, Smith GD, Schneider M, Minder C. Bias in meta-analysis detected by a simple, graphical test. Bmj Br Med J. 1997;315(7109):629-34.

31. Ding H. A systematic review and meta-analysis of the seroprevalence of toxoplasma gondii in cats in mainland China. Parasit Vectors. 2017;10(1):27.

32. Moher D, Liberati A, Tetzlaff J, Altman DG, Group TP. Preferred reporting items for systematic reviews and meta-analyses: the PRISMA Statement. Open Med. 2009:3(3):e123.

33. Guan WH, Yuan HC, Zhao GM, Yu JM, Yang QJ. Survey of schistosomiasis epidemic in dam circle marsh region. China Public Health. 1999;15(8):686-8.

34. Li JH, Wang TP, Xiao X, Wu WD, Lu DB, Fang GR, Cai W, Zheng J, Xu J, Wang RR. Cost-effectiveness of screening methods for determining the subjects of chemotherapy in areas with low endemic of schistosomiasis. Parasitoses Infect Dis. 2002;10(4):145-8.

35. Jin J, Pan X, An J, Tang J, Zhou Z, He L, Gao S, Chen D, Liu Y, Ruan B. Study on the correlation of detectable rates for schistosomiasis between the techniques of IHA and Kato- Katz in different endemic areas. J Trop Dis Parasitol. 2005;3(4):204-6.

36. Xiao X. Development and evaluation of a novel visually-read colloidal dye immunofiltration assay for rapid diagnosis of Schistosome Japonicum infection. University of Science and Technology of China; 2005.

37. Bao JG, Qiang GX, Deng YJ, Zhang R, Chen Y. Field comparison of four diagnostic methods for the detection of schistosomiasis. J Trop Dis Parasitol. 2006;4(1):33-4.

38. Yu JM, Vlas SJD, Jiang OW, Gryseels B. Comparison of the Kato-Katz technique, hatching test and indirect hemagglutination assay $(\mathrm{IHA})$ for the diagnosis of Schistosoma japonicum infection in China. Parasitol Int. 2007; 56(1):45-9.

39. Lin DD, Liu YM, Hu F, Tao B, Wang XM, Zuo XX, Li JY, Wu GL. Evaluation on application of common diagnosis methods for schistosomiasis japonica in 
endemic areas of China I Evaluation on estimation of prevalence of Schistosoma japonicum infection by IHA screening method. Chinese J Schistosomiasis Control. 2008;20:3.

40. Zhou YB, Yang MX, Tao P, Jiang QL, Zhao GM, Wei JG, Jiang QW. A longitudinal study of comparison of the Kato-Katz technique and indirect hemagglutination assay (IHA) for the detection of schistosomiasis japonica in China, 2001-2006. Acta Trop. 2008;107(3):251.

41. Zhong ZJ, Zeng XJ, Jiang YW, Hu WC, Lan WM, Zhang ZZ, Gao XJ, Xie F, Ge J, Lu SB. Serum epidemiological analysis of schistosomiasis in Poyang Lake residents. Exp Lab Med. 2009;27(2):141-2.

42. Hu G, Zhu CF, Li JX, Cai KP. Effect of project of diverting intermediate water to Xizha on Oncomelania snail and schistosome cercaria control in Junshan District. Chinese J Schistosomiasis Control. 2010;

43. Lin SY: Accuracy of diagnosis methods on Residents' Schistosome infection in Lake regions. Huazhong University of Science and Technology; 2010.

44. Liu L, Yang GJ, Zhu HR, Yang K, Ai L. Knowledge of, attitudes towards, and practice relating to schistosomiasis in two subtypes of a mountainous region of the People's republic of China. Infect Dis Poverty. 2014;3(1):16.

45. Huang XB, Su ZW, Feng DC, Fu Y, Hu CQ. Field application of Dot-ELISA for detection of circulating antigens of Schistosomiasis. Chinese J Schistosomiasis Control. 1994:6:391.

46. Song HT, Liang YS, Dai JR, Ji CS, Shen XH, Li LG, Yin F. Evaluation on dipstick dye Immuno-assay for screening chemotherapy targets of schistosomiasis in a lower endemic area. Chinese J Schistosomiasis Control. 2003;

47. Chen SR, Chen F, Zhou XN, Hong-Jun LI, Jacob P, Yang Z, Yuan-Lin LI. Comparison of aetiological and serological diagnosis methods in schistosomiasis mountainous endemic area. Parasitoses Infect Dis. 2007;

48. Wei H, Zhu YC, Liang YS, Dai JR, Ming X, Tang JX, Cao GQ, Hua WQ, Li YL, Zhong $Y$. Comparison of stool examination and immunodiagnosis for schistosomiasis. Chinese J Schistosomiasis Control. 2007:

49. Yu LL, Ding JZ, Wen LY, Lou D, Yan XL, Lin L, Lu SH, Lin DD, Zhou XN. Development of a rapid dipstick with latex immunochromatographic assay (DLIA) for diagnosis of schistosomiasis japonica. Parasit Vectors. 2011;4:157.

50. She YB. Comparison of four detection methods in areas of schistosomiasis transmission control. Exp Lab Med. 2015;2:237-8.

51. Wu ZD, Hu YL. Field application of McAb-Dot-ELISA for detection of circulating antigens of Schistosomiasis. J Nanchang Univ (Med Sci). 1992;(2):121-3.

52. Xu J, Chen NG, Feng T, Wang EM, Wu XH, Chen HG, Wang TP, Zhou XN, Zheng J. Effectiveness of routinely used assays for the diagnosis of schistosomiasis japonica in the field. Chinese J Parasitol Parasit Dis. 2007; 25(3):175.

53. Zhou YB, Yang MX, Wang QZ, Zhao GM, Wei JG, Peng WX, Jiang QW. Field comparison of immunodiagnostic and parasitological techniques for the detection of Schistosomiasis japonica in the People's Republic of China. Am J Trop Med Hyg. 2007;76(6):1138-43.

54. He JB, Li S, Chen JS, Wang XK. Using general control data to speculate the infection of schistosomiasis. J Trop Dis Parasitol. 2008;

55. Lin DD, Xu J, Liu HY, Zeng XJ, Liu YM, Xie SY, Hu F, Li JY, Huang MJ, Chen HG. Comparative evaluation of five test kits for antibody detection in Schistosoma japonicum endemic areas of Poyang Lake region. Chinese Parasitol Parasit Dis. 2010;28(6):439.

56. Cai YC, Xu JF, Steinmann P, Chen SH, Chu YH, Tian LG, Chen MX, Li H, Lu Y, Zhang LL. Field comparison of circulating antibody assays versus circulating antigen assays for the detection of schistosomiasis japonica in endemic areas of China. Part Fibre Toxicol. 2014;7(1):138.

57. Wang XH, Wu XH, Zhou XN. Bayesian estimation of community prevalences of Schistosoma japonicum infection in China. Int J Parasitol. 2006;36(8):895.

58. Zhu H, Cao C, Wang Q, Bao Z, Zheng H, Guo J. Study on reasons of underestimation of the number of Schistosoma japonicum infected persons in mountainous endemic regions. Chinese J Schistosomiasis Control. 2006;

59. Yang JZ: Analysis of the risk factors of schistosomiasis using multilevel models. Central South University; 2007.

60. Hua Y, Zhao ZY, Ren MY. Epidemiological surveillance and analysis on schistosomiasis in endemic areas of West Dongting Lake in 2006. Pract Prev Med. 2008;

61. Wang XH, Zhou XN, Vounatsou P, Chen Z, Utzinger J, Yang K, Steinmann P, Wu XH. Bayesian spatio-temporal modeling of Schistosoma japonicum prevalence data in the absence of a diagnostic 'gold' standard. PLoS Negl Trop Dis. 2008;2(6):e250.

62. Gao B, Huang XH, Li ZS, Fang ZJ. Surveillance Of Schistosomiasis In Jiahu Village Of Yugan County, Jiangxi Province, 2007. J Trop Med. 2009;96:75-6.
63. Tao B, Jiang QL, Luo CJ, Yin ZH, Wang JM. Endemic situation of schistosomiasis in Xingzi County. Chinese J Schistosomiasis Control. 2009:62-3.

64. Mao Y, Zhong B, Yang Y. Evaluation of water engineering project for schistosomiasis control in Meishan and Guanghan in Sichuan, 2007-2008. Parasitoses Infect Dis. 2010;

65. Zhou FC, Huang SX, Li QY, Luo HT, Zhou HM, Ying QB: Surveillance of schistosomiasis situation from 2005 to 2009. Chinese J Hyg Insecticides Equip 2010.

66. Shang-Yang SU, Xiang SW, Chen ZF, Liu XH. Surveillance of epidemic of schistosomiasis in Machuan village of Shishou city from 2005 to 2011. J Trop Med:2013.

67. Wu ZS, Tang M, Li HG, Xu L, Zhu HB, Fan JR, Mao P, Luo F, Huang Y, Zhang $Y$. Surveillance of schistosome antibodies in population in mountainous schistosomiasis endemic regions in China. Chinese J Schistosomiasis Control. 2013:25(2):133-6.

68. Dang H, Xu J, Li SZ, Cao ZG, Huang YX, Wu CG, Tu ZW, Zhou XN. Monitoring the Transmission of Schistosoma japonicum in Potential Risk Regions of China, 2008-2012. Int J Environ Res Public Health. 2014;11(2):2278.

69. Zhang $X$, Cui CX, Zhang WR, Wen XH, Ma N, Zou P, Tu ZW, Hu HH, Liu $X$, Cao CL. Efficacy of routinely used patterns for schistosomiasis diagnosis in lake and marshland regions. Chinese J Schistosomiasis Control. 2014:26(6):618-21.

70. Yu JM, de Vlas SJ, Yuan HC, Gryseels B. Variations in fecal Schistosoma japonicum egg counts. Am J Trop Med Hyg. 1999;20(1):370.

71. Chen LZ. Survey on the prevalence of schistosomiasis in villages with different levels of outbreaks. J Pathogen Biol. 2010;

72. Wu XH, Xu J, Li SZ, Huang YX, Wang TP, Wu CG, Huang XB, Feng M, Chen $Z$, Wang LY. Prediction and surveillance of schistosomiasis japonica in potential endemic areas in China, 2008. Chinese J Schistosomiasis Control. 2009:353-7.

73. Zhang YY, Luo JP, Liu YM, Wang QZ, Chen JH, Xu MX, Xu JM, Wu J, Tu XM, Wu GL. Evaluation of Kato-Katz examination method in three areas with low-level endemicity of schistosomiasis japonica in China: a Bayesian modeling approach. Acta Trop. 2009;112(1):16-22.

74. Engels D, Sinzinkayo E, Gryseels B. Day-to-day egg count fluctuation in Schistosoma mansoni infection and its operational implications. Am J Trop Med Hyg. 1996;54(4):319.

75. Lin DD, Liu YM, Hu F, Li YF, Tao B, Yuan M, Xie SY, Huang MJ, Jiang QL, Li JY. Evaluation on application of common diagnosis methods for schistosomiasis japonica in endemic areas of China. III. Analysis and evaluation of underestimation of prevalence of Schistosoma japonicum infection by routine Kato-Katz technique. Zhongguo Xue Xi Chong Bing Fang Zhi Za Zhi. 2011;23(6):642-7.

76. Zhu H, Yu C, Xia X, Dong G, Tang J, Fang L, Du Y. Assessing the diagnostic accuracy of immunodiagnostic techniques in the diagnosis of schistosomiasis japonica: a meta-analysis. Parasitol Res. 2010;107(5):1067-73.

77. Doenhoff MJ, Chiodini PL, Hamilton JV. Specific and sensitive diagnosis of schistosome infection: can it be done with antibodies? Trends Parasitol. 2004;20(1):35

78. Wu G. A historical perspective on the immunodiagnosis of schistosomiasis in China. Acta Trop. 2002;82(2):193.

Ready to submit your research? Choose BMC and benefit from:

- fast, convenient online submission

- thorough peer review by experienced researchers in your field

- rapid publication on acceptance

- support for research data, including large and complex data types

- gold Open Access which fosters wider collaboration and increased citations

- maximum visibility for your research: over $100 \mathrm{M}$ website views per year

At $\mathrm{BMC}$, research is always in progress.

Learn more biomedcentral.com/submissions 